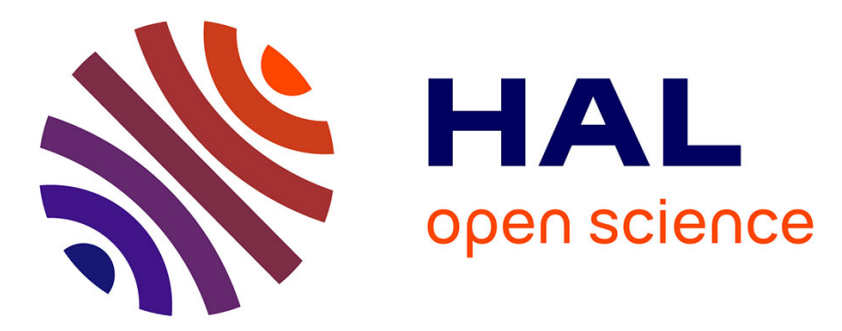

\title{
A laterally coupled UV210 polymer racetrack micro-resonator for thermal tunability and glucose sensing capability
}

R Castro-Beltrán, Nolwenn Huby, V. Vié, H Lhermite, Lionel Camberlein, Etienne Gaviot, Bruno Bêche

\section{To cite this version:}

R Castro-Beltrán, Nolwenn Huby, V. Vié, H Lhermite, Lionel Camberlein, et al.. A laterally coupled UV210 polymer racetrack micro-resonator for thermal tunability and glucose sensing capability. Advanced Device Materials, 2015, 1 (3), pp.80-87. 10.1080/20550308.2015.1133100 . hal-01249190

\author{
HAL Id: hal-01249190 \\ https://hal.science/hal-01249190
}

Submitted on 30 Dec 2015

HAL is a multi-disciplinary open access archive for the deposit and dissemination of scientific research documents, whether they are published or not. The documents may come from teaching and research institutions in France or abroad, or from public or private research centers.
L'archive ouverte pluridisciplinaire HAL, est destinée au dépôt et à la diffusion de documents scientifiques de niveau recherche, publiés ou non, émanant des établissements d'enseignement et de recherche français ou étrangers, des laboratoires publics ou privés. 


\title{
A laterally coupled UV210 polymer racetrack micro-resonator for thermal tunability and glucose sensing capability
}

\author{
R. Castro-Beltrán ${ }^{1}$, N. Huby' ${ }^{1}$, V. Vié1, H. Lhermite ${ }^{2}$ L. Camberlein ${ }^{3}$, E. \\ Gaviot $^{3}$, B. Bêche ${ }^{*}$ \\ ${ }^{1}$ Institut de Physique de Rennes, IPR UMR CNRS 6251, Université de Rennes 1, bât. 11E \\ Nanosciences, 263 avenue Général Leclerc, 35042 Rennes, France \\ 2 Institut d'Electronique et de Télécommunications de Rennes, IETR UMR CNRS 6164, \\ Université de Rennes 1, bât. 11B, 263 avenue Général Leclerc CS 74205, 35042 Rennes Cedex, \\ France \\ ${ }^{3}$ Laboratoire d'Acoustique de l'Université du Maine, LAUM UMR CNRS 6613, Université du \\ Maine, Avenue Olivier Messiaen, 72085 Le Mans Cedex 9, France
}

*Dr. R. Castro-Beltrán is now with the Centro de Investigaciones en Óptica A.C, Loma del Bosque 115, Col. Lomas del Campestre, C.P. 37150 León, Guanajuato, México

"Corresponding author, email bruno.beche@univ-rennes1.fr

\begin{abstract}
We report and demonstrate the feasibility of a laterally coupled racetrack microresonator based on UV210 photoresist to act as a thermal and glucose sensor. The large thermo-optic coefficient and the detection principle based on the interaction of the evanescent field with different glucose concentrations demonstrate that this sensor displays high sensitivity on detection properties. Deep-UV lithography procedures allow us to develop a laterally coupled microresonator with submicrometer patterns. The thermo-optic response of the racetrack microresonator is interrogated by using a $\mathrm{NiCr}$ alloy tip positioned on the top of the device. Temperatures ranging between 19 and $33^{\circ} \mathrm{C}$ yield a red shift of the resonant wavelength with a linear sensitivity of $220 \mathrm{pm}^{\circ} \mathrm{C}^{-1}$. Additionally, the thermal tunability is successively demonstrated by covering the resonator with DI water. A blue shift of the resonant wavelength is obtained with a linear sensitivity of $200 \mathrm{pm}^{\circ} \mathrm{C}^{-1}$. The resonance optical properties under this top cladding conditions lead a Q-factor of 4000 with a finesse of 5.7 .

Glucose homogeneous sensing capability is also experimentally demonstrated. Different concentrations of glucose solutions result in a red shift of the resonant wavelengths with a linear sensitivity of $280 \mathrm{pm} /(\mathrm{mg} / \mathrm{ml})$. Finally, these results validate the laterally coupled racetrack microresonator as an operative photonic component for integrated optical devices such as optical filters applied on telecommunication, or transducer components devoted to assess biochemical interactions.
\end{abstract}

Keywords Organic, Deep UV polymer, Integrated photonics, Micro-resonators, Thermally tunable and Glucose sensors 


\section{Introduction}

Both manufacturing processes and inter-cellular signal transductions demand an effectual next generation of chip-submicroscale sensors with the aim of respectively achieving effective thermomechanical monitoring under hostile working conditions and a better understanding of multiple signaling transductions ${ }^{1-3}$. Although significant advances have been attained in the micro fabrication of novel sensor technologies, low cost fabrication, integration with existing technologies, portability, robustness and customizability remain as strong requirements demanded by the commercial sector. Additionally, the sensor platform requires an effective capability to process and differentiate multivariable data, which calls for arrays of different sensor configurations integrated into a single chip ${ }^{2}$. Furthermore, the submicrometer size condition is essential since small devices are able to respond quickly to environment changes and feature enhanced sensitivity properties. Regarding the above-mentioned considerations, optical sensors are good candidates for telecom and biosensing applications, because they are easy to fabricate and to integrate with existing technologies ${ }^{3-8}$. In the domain of optical integrated (OI) devices, polymer materials especially offer various advantages with respect to others, such as low production cost, ease of manipulation and fabrication, high thermo-optic coefficients and high coupling efficiency to optical fibers ${ }^{3,9,10}$. The intrinsic high thermo-optic property presented on OI devices built on polymeric materials turns them attractive for data communications and multivariable biochemical treatments because they have an active region that is either very effective, sensitive or both ${ }^{11,12}$. Additionally, this property and the submicrometer size make them feasible optical devices for characterizing and monitoring novel configuration of thermal flow microheaters ${ }^{13}$.

On the other hand, for biological and chemical detection, polymer materials can be easily surface-activated, wherewith high specificity and selectivity can be achieved ${ }^{3,8,14}$. Currently, the most studied OI sensors are based on Mach-Zehnder interferometers, grating sensors, surface plasmon sensors and optical microresonators $2-8,14$. There is significant attention to optical microresonators for manufacturing processes and for biosensing applications, mainly due to their structural simplicity, compactness in size, simplicity in fabricating and their capability to biochemically target their surface and follow the biorecognition by means of the extension of their evanescent field. Besides the fact that relevant advantages can be adjusted depending on the application through the relationship fabrication protocol-design, several shapes such as microdiscks, microracetracks and microspheres may be considered ${ }^{15-17}$.

Polymeric microresonators have been successfully used as: strain sensors, laser microcavities, modal filters, and as optical temperature and glucose sensors ${ }^{11,18-20}$. In this work we present the viability of a specific microresonator, based on a racetrack shape and on UV210 photoresist, as a temperature and glucose sensor. Recently, UV210 photoresist has been used as single mode rib waveguides ${ }^{21}$ and submicrometer 2D-resonators ${ }^{15}$, demonstrating the high capability of the polymer for photonic miniaturization. Then, the design and the overall fabrication and optical characterization of a cascade of multiple microresonators acting as modal filters have been presented ${ }^{16}$. In order to demonstrate the complete feasibility of the UV210 photoresist to support OI devices, this paper aims to present the first developments of this polymeric microracetrack for temperature and homogeneous glucose sensing. Section II briefly describes the design and fabrication of such a polymeric microracetrack. Section III presents the experimental protocol for optical characterization of the devices under test. Section IV and section V respectively present the validation of the photonic device acting as temperature and homogeneous glucose sensor. 


\section{Microracetrack design, fabrication and device visualization}

An OI device based on a racetrack microresonator consists of two main parts: the bus optical waveguide and the resonator with an elongated coupling zone. The bus optical waveguide works as an input/output optical waveguide channel, the resonator being where the coupled optical wave makes multiple round trips. It is known that the most important optical parameters that describe the response and behavior of a microresonator are the free spectral range FSR, the full width half maximum FWHM, the quality factor Q and the finesse F. Enhancing these properties depends essentially on fabrication protocols together with the selected resonator design. Particularly, an improvement on the coupling mechanism makes better the performance of the microresonators: this can be achieved either by selecting alternate photonic circuit design strategies such as vertically coupled microresonators (which implies more fabrication costs), or simply by elongating the coupling region of the microresonator ${ }^{14,16}$. In this work, we have focused on the resonator based on UV210 photoresist, and developed through deep UV (DUV) lithography, procedures yielding a racetrack-shaped geometry with apt lateral coupling mechanisms with respect to the bus optical waveguide.

\subsection{Microracetrack design}

As illustrated in figure 1, the microracetrack is coupled with two straight slowly tapered optical waveguides, mainly assuring the single mode operation of the OI device and, moreover, the efficient power-coupling due to the optical parameters considered in this study ${ }^{22}$. The distance separating the bus optical waveguides from the optical microresonator is named gap size. In a microresonator configuration, this pivotal geometrical condition determines the coupling strength required between both elements, which is directly transferred and displayed on the resonance transmission with poor or excellent properties.

\section{Figure 1}

For laterally coupled OI microresonators, the development of devices with a gap in the subwavelength order is not an easy task. Our device was fabricated following the DUV lithography procedures and considering the UV210 photoresist handling conditions, which enable to develop OI devices in the submicrometer order ${ }^{16,23}$. More specifically, the $400 \mathrm{~nm}$ gap between the cavity and the rib waveguides ensures an apt optical coupling via an evanescent field. This gap value overcomes those discussed in previous publications, where we also observed clear resonance transmission ${ }^{15,16}$. The $\mathrm{R}=50 \mu \mathrm{m}$ radius and the $\mathrm{Lc}=60 \mu \mathrm{m}$ coupling length are both main features characterizing the geometry of a racetrack. Furthermore, the alignment and the parallelism variation between the rib optical waveguide and the elongated zone in the resonator are crucial to get a high coupling efficiency within the OI device.

On the other hand, although the OI device has a doubly-coupled configuration, the optical characterization and the experimental protocol are based on a directional coupler where the micoresonator is singly-coupled through an optical waveguide. Considering Fig. 1, the port in and port out are respectively the ports at which light is injected and the optical spectrum is transmitted. Regularly, an optical add-drop filter is traditionally used to insert or extract specific wavelength channels in a wavelength division multiplexed (WDM) system and has become a fundamental building block for optical communication systems. Nevertheless, this work focuses 
on sensing capabilities, thus all consecutive optical characterizations will carry out through this singly-coupled configuration.

Details about the main parameters are discussed below with relevant images obtained by scanning electron microscopy (SEM) and a micro beam profile (MBP) system.

\subsection{Fabrication and optical visualization: DUV lithography processes, SEM and MBP images}

Devices are fabricated through DUV lithography, the whole process being performed in a cleanroom. The starting point of the fabrication is a commercial silicon wafer covered with a 1.5 $\mu$ m-thick thermal $\mathrm{SiO}_{2}$ layer. A $1 \mu \mathrm{m}$-thick layer of the UV210 polymer is spin-coated on the $\mathrm{SiO}_{2} / \mathrm{Si}$ substrate. After softbaking procedures, the microresonators patterns are transferred onto the UV210 polymer layer. This is immediately followed by the post exposure bake (PEB) procedure in order to complete the cross-linked polymeric mechanism. Before developing, the sample is cooled down, which enhance the quality of the patterning of the devices. Then, the sample is immersed in the developing solution, and the submicrometer patterns are revealed. Finally, the last step consists in fully cross-linking the polymer layer by means of a curing process, also referred to a hard bake step. This process is realized in an oven with temperatures ranging from $100{ }^{\circ} \mathrm{C}-150{ }^{\circ} \mathrm{C}$ over $24 \mathrm{~h}$. The importance of this final step is critical on the fabrication of these devices because with unsuitable hard bake steps poor conditions of crosslinking are achieved favoring significant sorption of water molecules through the polymer matrix which in addition, changes the rigid conditions of the devices ${ }^{24,25}$.

For OI devices based on polymeric materials and fabricated through optical lithography procedures, it is decisive to assess the main structural qualities, such as surface conditions of the photonic devices before optical characterization. Sidewall roughness yields an untoward strong effect on the optical features of these OI devices due to the degree of light scattering: unfortunately, with devices fabricated under these conditions, roughness on the vertical sidewalls of the waveguide is unavoidable ${ }^{26}$. Scanning electron microscopy and Micro Beam Profiler (MBP) images of the device have been systematically recorded, and the main results are gathered consecutively on Fig. 2. The corresponding MBP images are taken by considering normal environmental conditions with air as upper cladding.

\section{Figure 2}

Figure $2 a$ presents a top-view perspective of the submicrometer optical racetrack microresonator. The OI device is well defined presenting a radius of $\mathrm{R}=50 \mu \mathrm{m}$, a width of $\mathrm{w}=5 \mu \mathrm{m}$ and a coupling length of $\mathrm{Lc}=60 \mu \mathrm{m}$. The measurement of the gap width leads to a mean value of 400 $\mathrm{nm}$ with a standard deviation calculated for several measurements along the coupling length of $30 \mathrm{~nm}$, showing the high accuracy of the DUV lithography method and the degree of parallelism between both the bus and the coupling region in the device. Finally, the light propagation along the bus waveguide and the loop of the racetrack microresonator is depicted in Fig. $2 b$. It also displays the coupling light along the coupling region, thus confirming the evanescent coupling between rib waveguides and microresonators. Such images reproduce the design conditions while also demonstrating the suitable accuracy of DUV optical lithography to fabricate photonic structures. 


\section{Optical characterization of the photonic devices under test: experimental protocol}

Optical racetrack microresonators are then characterized using the experimental platform presented in Fig. 3.

\section{Figure 3}

The device is characterized under two optical broadband sources. The first one is centered at 845 $\mathrm{nm}$ with an FWHM of $30 \mathrm{~nm}$, and the second one at $1025 \mathrm{~nm}$ with an FWHM of $45 \mathrm{~nm}$. In waveguide sensors whose detection mechanism is based on the extended evanescent field, one of the key parameters weighing on the performance of the device is precisely the penetration depth of the field highly depending on the wavelength source value. Considering the laboratory conditions, we choose to carry out the homogeneous glucose sensing by way of the broadband source centered at $1025 \mathrm{~nm}$, so as to maximize the penetration depth. The transverse electric TE polarization of both sources is controlled by combining a polarizer and a half-wave plate. The beam is then focused and coupled with the photonic devices through the port in of the rib waveguide. The input and output beams are respectively guided with two X20 microscope objectives. The device and the microscope objectives are positioned on piezoelectric nanopositioners for better stability and injection facilities. The beam at the output port of the rib waveguide is monitored with an infrared (IR) camera and an optical powermeter. Once the maximum power is obtained, the optical powermeter is replaced by an optical spectra analyzer (OSA Ando AQ-6315E) for resonance detection analysis.

The source used for the resonance measurements is a broadband laser: therefore, the effective group index $\mathrm{n}_{\mathrm{eff}}^{\mathrm{grp}}$ instead of the refractive index of the materials for the optical resonances linked to each quantified WGMs has to be considered. This effective index takes into account the group velocity vg of the light wave package, which is also more relevant for broadband sources. Due to the physical principle of the WGMs resonances and the optical properties, these modes occur at regular spectral intervals called the Free Spectral Range (FSR) defined as $\Delta \lambda=\lambda_{0}^{2} /\left(\mathrm{P} \cdot \mathrm{n}_{\mathrm{eff}}^{\mathrm{grp}}\right)$, with $\lambda_{0}$ the excitation laser wavelength in vacuum and $P$ the geometrical perimeter of the MR under test.

Figure 4 is a typical normalized spectral response from a microracetrack resonator carried out at room temperature $\left(20^{\circ} \mathrm{C}\right)$ and the air as upper cladding condition.

\section{Figure 4}

The normalized FFT response, presented in Fig. 4b, corroborates the theoretical approach with the experimental one. Regarding the geometrical conditions of the OI device and using $n_{U V 210}=$ 1.556 at $850 \mathrm{~nm}$ (Cauchy law), FSR values of 1.02-1.13 nm are expected for $\lambda_{0}$ ranging from $820 \mathrm{~nm}$ to $860 \mathrm{~nm}$. A possible minor difference between the theoretically and experimentally

obtained values can be explained by considering the dispersion of the $n_{\text {eff }}^{g r p}$ quantity inherent to the use of optical large bandwidth sources. Through Lorentzian fitting, values of $0.27 \mathrm{~nm}$ for FWHM at $\lambda=853.77 \mathrm{~nm}$, Q-factor of 3100 and F of 4.7 are calculated. This result is 2 times 
higher in comparison with the previous one obtained for devices with one resonant structure developed with this photoresist, which is mainly due to the reduced gap size value characterizing this racetrack microresonator ${ }^{16,27}$.

\section{Temperature sensor based on racetrack microresonator: test results}

The combination of the intrinsic high thermo-optic property with the microresonator featured as a transducer system could enable the use of such structure as a tunable optical filter. It is the aim of this section to assess the thermal tunability and consider the feasibility of the polymeric UV210 optical racetrack microresonator as a thermal sensor. The optical bench depicted in Fig. 4 is modified by including a heating resistant tip of $\mathrm{Ni}-\mathrm{Cr}$ alloy on the top of the device under test. The relative position of the heating tip is controlled using a xyz micrometric mount, while the tilted images of the interaction between the de OI device and the tip are recorded with a webcam. Before carrying out the measurements of thermal tunability, the calibration curve of the thermal alloy Ni-Cr tip is obtained by considering a relevant NTC thermistor temperature response as a function of the current circulating through the tip. Once the calibration curve is defined, the experimental protocol of the thermal response is realized by covering the device respectively with air and deionized (DI) water.

Figure 5 presents the plot of the measured resonant wavelength evolution taken with air as upper cladding. Their corresponding Lorentz fittings as the temperature of the thermal tip is increased from $19{ }^{\circ} \mathrm{C}$ to $33{ }^{\circ} \mathrm{C}$ are also presented. By selecting a resonance peak at $847.71 \mathrm{~nm}$ the OI device follows the temperature inputs with high accuracy. Moreover, Fig. $5 b$ presents the thermal relationship and the specific hysteresis curve of the OI device.

\section{Figure 5}

With temperature below $30{ }^{\circ} \mathrm{C}$, a red-shift linear trend is observed in Fig. $5 \mathrm{~b}$ for this polymeric UV210 racetrack microresonator with a rate of thermal sensitivity averaging $220 \pm 11.6 \mathrm{pm}^{\circ} \mathrm{C}^{-1}$. Nevertheless, a slight tendency to saturate the thermo-optic response is observed when the temperature is increased above $30^{\circ} \mathrm{C}$. Such a nonlinear response shows that for this regime the temperature is enough to change the density of the UV210 polymer which directly impinges on the thermo-optic effect of the polymer $(\Delta n / \Delta T)$.

Fig. $5 b$ shows the comparison between the corresponding ascending and descending slopes, which respectively represent the heating/cooling of the OI device under test with the thermal tunability signals. This behavior demonstrates the high reproducibility and controllability of the spectral responses with a minor lagging effect between them. These results highlight the mean values of four ascending and descending measurements with intervals of 5 minutes between each point for stability on the signal. By taking into account the refractive index for TE mode $n_{U V 210}=$ 1.556 and the assessed thermal sensitivity, the thermo-optic coefficient of the UV210 photoresist is calculated as $\Delta n / \Delta T \approx-2.3 \times 10^{-4}{ }^{\circ} \mathrm{C}^{-1}$, which is in good agreement with known values for polymers ${ }^{11,28,29}$.

Additionally, the blue-shift thermal tunability of the optical racetrack microresonator is demonstrated by varying the top cladding conditions. Figure 6 shows the thermal tunability response by covering the OI devices with DI water. The blue-shift observed on the thermal tunability of the resonance peak cannot be explained by considering just the thermo-optic effect 
as mentioned above. This linear variation is due to the combination of the refractive indices and volume changes of the racetrack microresonator ${ }^{3,30}$.

Moreover, under these conditions, the experimental curves no longer present the slight saturation phenomenon observed when air was the upper cladding. Such a behavior is mainly explained with the compared heat transfer capability of both claddings: Indeed, the air being quite less dense than DI water, both convection and conduction superimposed mechanisms entail a faster thermo-optic saturation effect in the OI device.

\section{Figure 6}

The stability presented on the experimental results depicted in Fig. $6 \mathrm{~b}$ demonstrates that an efficient and complete fabrication protocol for our OI polymeric devices was good enough to avoid misunderstandings on the transmission properties of the OI device due to water absorption. Here, we have to highlight the importance of the hard bake exposure in the fabrication steps for better fully cured devices. When this last step takes place, these polymeric materials have a very high level of crosslinking which leave little space in between the molecular chains for water tBesideo be absorbed.

A new value of Q- 4000 at $\lambda=852.575 \mathrm{~nm}$ is calculated due to refractive index symmetric conditions ${ }^{31}$. Furthermore, important features arising from this result are that for an expected homogeneous sensing, this blue-shift must be considered to avoid any misunderstanding in the data interpretation as the analyte should be recognized by the surface of the resonator.

On the other hand, for the purpose of the article, these results represent a very significant aspect in the evolution of the UV210 photoresist as a multifunctional photonic submicrometer material. Therefore, red and blue-shift behaviors on the resonance peak allow us to consider the racetrack microresonator as a tunable device by alternating with different upper cladding conditions, e.g., DI water and air.

\section{Glucose sensing: demonstration of homogeneous sensing}

- In the domain of biochemical sensors, microresonators used as OI devices present valuable advantages in comparison to other detection techniques whose detection principles are based on electrical, magnetic and mechanical signals. Mainly, these OI devices are constituted with optical waveguides with cavity shapes confining discrete sets of wavelengths: their optical biointerrogation hinges on the extension of an evanescent-field. The wavelength shifts essentially happen because the bulk refractive index of the OI device change ${ }^{2,32}$. In the bulk refractive index sensing, there are two sensing mechanisms, with homogeneous and surface sensing. In both mechanisms the biochemical solution serves as upper cladding layer of the OI device wherewith, different concentrations change the effective refractive index of the guided mode. In homogeneous sensing, the molecules in the solution contribute to the refractive index change: However, in this measuring scheme it is not possible to provide specificity. On the other hand, in the surface sensing mechanism are created intentionally binding sites on the surface to attract specific analytes within the aqueous solution. This route allows adsorption features and provides a way to selectively detect specific analytes ${ }^{33}$. It is the aim of this work to present and demonstrate the homogeneous sensing capability of the racetrack microresonator based on UV210 photoresist by measuring different concentrations of glucose solutions in DI water in the static mode $(0,0.1,0.3,0.6$ and $1 \mathrm{mg} / \mathrm{ml})$. 
Glucose is a prominent analyte with a very high demand, because its control is of vital importance in diabetic patients, and a glucose sensor is an integral part of an artificial pancreas with its controlled insulin release. Optical MRs sensors are presented such as an excellent and convenient opportunity for noninvasive glucose monitoring procedures: it is the same with other biochemical entities as relevant development would allow either automated control on biological processes or on-line control in the industry applications, e,g., agriculture and food.

Previously, the thermo-optic effect on this OI device based on polymeric materials has been demonstrated: furthermore, when sensing measurements are carried out in a solution, a given temperature variation is associated with a blue-shift which can influence the data obtained from biorecognition. Therefore, an apt temperature control of the device is mandatory, and the setup presented in Fig. 3 is modified and replaced by a thermostatic mount on which the OI device is positioned, integrating a thermistor for temperature measurement and a thermoelectric cooler connected to a PID temperature servo system. All measurements are performed at $20{ }^{\circ} \mathrm{C}$ and with DI water as reference and as upper cladding. Then, glucose solutions with different concentrations are spread on the microresonator with a drop volume high enough to avoid evaporation: before measurements, they are allowed to rest between 3 to 5 minutes for thermal equilibrium. After each measurement, the OI device is rinsed with DI water and dried with compressed air.

Compared to the first study devoted to a thermal measurement sensor whose upper cladding was air, the change of reference for this new glucose application detection will change slightly our reference in our two separate applications regarding thermal and glucose detections. It is clear that considering the reference with the upper cladding as pure water compared to the air on our protocol increases significantly the efficiency of the optical coupling between waveguide and the micro-resonator which is governed by the length of the lateral evanescent queue wave as $\mathrm{d}=\frac{\lambda_{0}}{2 \pi}\left(\mathrm{n}_{\text {eff }}^{2}-\mathrm{n}_{\text {clad }}^{2}\right)^{-1 / 2}$. With water as upper cladding, in our fixed configuration (geometry of the resonators, fixed wavelength and size value of the core waveguide/resonator), such a length will increase by a factor 1.5 then modifying slightly the selectivity in the spectral response. However this should be strictly devoid of any impact on the glucose sensor application, because both applications are intrinsically decorrelated (pure thermal measurement and glucose detection). The reference for the whole glucose detection is the set waveguide/resonator covered with a pure water upper cladding.

Figure 7 presents the measured wavelength resonance shift as a function of glucose concentration.

\section{Figure 7}

The resonant wavelength shift for glucose sensing depicted in Fig. $7 b$ shows off the reproducibility of the measurements and the linear response with a rate of glucose sensitivity averaging $287 \pm 45 \mathrm{pm} /(\mathrm{mg} / \mathrm{ml})$. The two experimental curves, referred to as $I$ and $I I$, in Fig. $7 b$ were operated consecutively and under the same protocol and experimental conditions. In this plot, we highlight the uncertainties of both measurements $I$ and $I I$ which correspond to five acquired samples for each glucose concentration: moreover, we emphasize the high level of reproducibility of the two independent obtained measurements. Besides the high reproducibility of these results, the two curves of Fig. $7 b$ show the high stability of the device to support 
measurements based on water with solutes without possible swelling trends on the optical waveguides constituting the OI device.

Since the resolution of the OSA is fixed at $0.1 \mathrm{~nm}$, with our protocol of glucose measurement with a thermal stabilization activated and set at a temperature $20^{\circ} \mathrm{C}$, we did not visualize any noise at a fixed glucose concentration and then a shift of the resonance peaks. As regards the lower limit of detection of such sensors devoted to low glucose detection concentrations ranging between [0-1] $\mathrm{mg} / \mathrm{ml}$, for typical glucose concentration values considered within human cells, we manage to detected a lower limit concentration down to $0.04 \mathrm{mg} / \mathrm{ml}$ corresponding to a relative change about $10^{-5}$ on the value of the optical refractive index of the glucose solution.

Such experimental data demonstrate the capability of this OI device based on UV210 photoresist to achieve homogeneous biorecognition measurements: moreover, its linear sensitivity is in the same order as that of published performances of polymeric microresonators for glucose homogeneous sensing. Finally, through these results we can conclude that after the first set of measurements, the properties on the transmission spectrum return to their initial conditions free of possible history effects on the signal which demonstrates the homogeneous sensing mechanisms of the experiments.

\section{Conclusions}

An integrated UV210 racetrack microresonator is fabricated, optically characterized and assessed for thermal tunability and for glucose homogeneous sensing measurements. An efficient fabrication process by means of deep UV lithography allows shaping a laterally coupled racetrack microresonator with adequate quality: this enables us to present the device as a chipscale integrated system with cogent properties to be used for thermomechanical monitoring and for biochemical sensing. The structural qualities such as shape, dimensions and surface conditions of the racetrack microresonator are characterized by scanning electron microscopy that confirms the sharp accuracy of the lithography technique to transfer submicrometer patterns onto the UV210 photoresist. A systematic optical characterization and FFT analysis of the transmission signals of the OI device under test show a set of resonant wavelengths with excellent agreement on the optical parameters with respect to those theoretically expected, with a calculated Q-factor of 4000 in DI water.

The possibility of tuning the set of resonant wavelengths by handling the thermo-optic effect of the UV210 photoresist is demonstrated by means of changing the temperature of a Ni-Cr alloy tip positioned on the top of the racetrack microresonator surface. Then, a linear thermal red-shift with sensitivity about $200 \mathrm{pm}^{\circ} \mathrm{C}^{-1}$ is obtained. Moreover, varying the top cladding conditions as the device is covered with DI water yields an effective tuning of the resonant wavelengths with shorter wavelengths (blue-shift). This linear blue-shift behavior is due to the combination of the refractive indices and the volume of the racetrack microresonator. Therefore, both red and blueshift variations presented on the resonance peak support the conclusion that the racetrack microresonator can be used as a tunable device by alternating top cladding conditions. Additionally, the homogeneous optical transduction mechanism is demonstrated by investigating with glucose on the top of the racetrack microresonator. Different concentrations lead to a shift of the resonant wavelengths by means of changing the analyte medium's refractive index. This shift shows off a linear response with a sensitivity of about $280 \mathrm{pm} /(\mathrm{mg} / \mathrm{ml})$. 
Finally, this work demonstrates that laterally coupled UV210 racetrack microresonators, fabricated through deep-UV lithography procedures, are valuable candidates for thermomechanical monitoring and for homogeneous glucose sensing. Furthermore, with such capabilities, a racetrack microresonator, acting as a transducer component in an optofluidic cell, could suitably be used in many areas, such as drug synthesis and environmental monitoring.

\section{Acknowledgements}

This work was supported by Region Bretagne and CNRS. The authors thank J. Le Lannic from CMEBA with the Rennes 1 University for SEM work, F. Artzner for helpful discussions on chemical aspect, S. Bouhier and L. Frein for technical support, as well as D. Torres from Centro de Investigaciones en Óptica for the realization of 3D-graphics.

\section{References}

1. X. Zhang and X. Li: "Design, fabrication and characterization of optical microring sensors on metal substrates", J. Micromech. Microeng., 2007, 18, 1-7.

2. H. K. Hunt and A. M. Armani: "Label-free biological and chemical sensors", Nanoscale, 2010, 2, $1544-1559$.

3. C. Delezoide, M. Salsac, J. Lautru, H. Leh, C. Nogues, J. Zyss, M. Buckle, I. Ledoux-Rak and C.T. Nguyen: "Vertically Coupled Polymer Microracetrack Resonators for Label-Free Biochemical Sensors", IEEE Photon. Technol. Lett., 2012, 24, 270-272.

4. P. Rivolo, F. Michelotti, F. Frascella, G. Digregorio, P. Mandracci, L. Dominici, F. Giorgis and E. Descrovi: "Real time secondary antibody detection by means of silicon-based multilayers sustaining Bloch surface waves", Sensors and Actuators B: Chemical, 2012, 161, 1046-1052.

5. A. Sinibaldi, N. Danz, E. Descrovi, P. Munzert, U. Schulz, F. Sonntag, L. Dominici and F. Michelotti: "Direct comparison of the performance of Bloch surface wave and surface plasmon polarition sensors", Sensors and Actuators B: Chemical, 2012, 174, 292-298.

6. M.A. Santiago-Cordoba, M. Cetnikaya, S.V. Boriskina, F. Vollmer and M.C. Demirel: "Ultrasensitive detection of a protein by optical trapping in a photonic-plasmonic microcavity”, J. Biophotonics, 2012, 5, 629-638.

7. M.A. Santiago-Cordoba, S.V. Boriskina, F. Vollmer and M.C. Demirel: "Nanoparticle-based protein detection by optical shift of a resonant microcavity”, Appl. Phys. Lett., 2011, 99, 07370.1-3.

8. K. De Vos, I. Bartolozzi, E. Schacht, P. Bienstman and R.Baets: "Silicon-on-Insulator microring resonator for sensitive and label-free biosensing", Opt. Express, 2007, 15, 7610-7615.

9. B. Bêche, N. Pelletier, E. Gaviot, R. Hierle, A. Goullet, J. P. Landesman, and J. Zyss: "Conception of optical integrated circuits on polymer", Microelectron. J., 2005, 37, 421-427.

10. T. Grossmann, T. Wienhold, U. Bog, T. Beck, C. Friedmann, H. Kalt and T. Mappes: "Polymeric photonic molecule super-mode lasers on silicon", Light Sci. Appl., 2013, 2, 1-4.

11. P. Regoliosi, M. Guehl, G. Scarpa, P. Lugli, L. Persano, P. Del Carro, A. Camposeo, R. Cingolani, D. Pisignano, S. Bietti, E. Grilli and M. Guzzi: "Thermal tunability of monolithic polymer microcavities", Appl. Phys. Lett., 2008, 92, 253310-1-253310-3.

12. D. Hohlfeld and H. Zappe: "An all-dielectric tunable optical filter based on the thermo-optic effect", J. Opt. A: Pure Appl. Opt., 2004, 6, 504-511.

13. J. T. W. Kuo, L. Yu and E. Meng: "Micromachined Thermal Flow Sensors - A Review", Micromachines, 2012, 3, 550-573.

14. C. Delezoide, C. Noguès, R. Castro, J. Lautru, M. Beckle, I. Ledoux-Rak, J. Zyss and C. T. Nguyen: “DualPolarization Optofluidic Biodetection Based on Polymer Microring Resonators", Proc. IEEE Lasers and ElectroOptics Europe (CLEO EUROPE/IQEC), 2013 Conf. on and International Quantum Electronics ConferenceMunich.

15. D. Pluchon, N. Huby, H. Lhermite, D. Duval, and B. Bêche: "Investigation of fabrication and resonant optical coupling in various 2D micro-resonator structures in a UV210 polymer", J. Micromech. Microeng., 2012, 22, 1-8. 
16. R. Castro-Beltrán, N. Huby, G. Loas, H. Lhermite, D. Pluchon and B. Bêche: "Improvement of efficient coupling and optical resonances by using taper-waveguides coupled to cascade of UV210 polymer microresonators", J. Micromech. Microeng., 2014, 24, 1-7.

17. D. Pluchon, N. Huby, A. Moreac, P. Panizza and B. Bêche: "From Fabrication to Characterization of 3D Organic Microresonators: A Complementary Alliance of Microfluidics and Optics", Advances in Optical Technologies, 2012, 2012, 1-7.

18. B. Bhola, H-C Song, H. Tazawa and W. H. Steier: "Polymer Microresonator Strain Sensors", IEEE Photon. Technol.Lett., 2005, 17, 867-869.

19. S. Lozenko, N. Djellali, I. Gozhyk, C. Delezoide, J. Lautru, C. Ulysse, J. Zyss and M. Lebental: "Enhancing performance of polymer-based microlasers by a pedestal geometry", J. Appl. Phys., 2012, 111, 103116-1-1031162.

20. S-Y Cho and N. M. Jokerst: "A Polymer Microdisk Photonic Sensor Integrated Onto Silicon", IEEE Photon.Technol. Lett., 2006, 18, 2096-2098.

21. D. Duval, H. Lhermite, C. Godet, N. Huby and B. Bêche: "Fabrication and optical characterization of submicronic waveguide structures on UV210 polymer", J. Opt., 2010, 12, 1-6.

22. T. Bec, S. Schloer, T. Grossmann, T. Mappes and H. Kalt: "Flexible coupling of high-Q goblet resonators for formation of tunable photonic molecules", Opt. Express, 2012, 20, 22012-22017.

23. P. B. Sahoo, R. Vyas, M. Wadhwa and S. Verma: "Progress in deep-UV photoresist", Bull. Mater. Sci., 2002, 25, 553-556.

24. http://www.microchemicals.com/technical_information/hardbake_photoresist.pdf

25. K. Jonghyun, K. Il, C. Yongwon and P. Kyung-Wook:" Studies on the Polymer Adhesive Wafer Bonding Method Using Photo-Patternable Materials for MEMS Motion Sensors Applications", IEEE Trans. Compon. Packag. Manuf. Technol., 2012, 2, 1118-1127.

26. S. C. Hagness D. Rafizadeh, S. T. Ho and A. Taflove: "FDTD Microcavity Simulations: Design and Experimental Realization of Waveguide-Coupled Single-Mode Ring and Whispering-Gallery-Mode Disk Resonators", IEEE J. Lightwave Technol., 1997, 15, 2154-2165.

27. C-Y Chao, W. Fung and L. J. Guo: "Polymer Microring Resonators for Biochemical Sensing Applications", IEEE J. Sel. Top. Quantum Electron., 2006, 12, 134-142.

28. Z. Zhang, P. Zhao, P. Lin and F. Sun: "Thermo-optic coefficients of polymers for optical waveguide applications", Polymer, 2006, 47, 4893-4896.

29. M. S. Nawrocka, T. Liu, X. Wang and R. R. Panepucci: "Tunable silicon microring resonator with wide free spectral range", Appl. Phys. Lett., 2006, 89, 071110-1-071110-3.

30. Y. Sanogo : "Conception et fabrication de capteurs et de leur technique d'interrogation pour des applications dans les domaines de la santé et de l'environnement", $\mathrm{PhD}$ thesis, Ecole Normale Superieure de Cachan, France, 2012.

31. H. Kogelnik and V. Ramaswamy: "Scaling Rules for Thin-Film Optical Waveguides", Appl. Opt., 1974, 13, 1857-1862.

32. W. Bogaerts, P. De Heyn, T. V. Vaerenbergh, K. DeVos, S. K. Selvaraja, T. Claes, P. Dumon, P. Bienstman, D.V. Thourhout and R. Baets:"Silicon microring resonators", Laser Photonics Rev., 6, 47-73.

33. Chao C-Y: "Polymer Microring resonator and its application as a biosensor", $2005 \mathrm{PhD}$ Dissertation University of Michigan, MT. 


\section{Figure and table captions}

Figure 1. Schematic representation of racetrack microresonator. Parameters $R, L c$ and $g$ represent respectively the radius, the coupling length and the distance between the rib waveguides and the microresonators.

Figure 2. Visualization of the structural details of the device under test through $a$ SEM and $b$ MBP system. The MBP images are taken under normal environmental conditions: air as upper cladding.

Figure 3. Experimental setup. Two broadband sources are used to optically characterize the sensor capability of the OI device. XYZ displacement mounts are used to control the NIR objectives (x20) and the device positions. The injection, propagation and detection are achieved through an IR camera, an optical powermeter and an MBP system. Optical resonances are finally analyzed with an OSA. The thermo-optical response of the device is characterized by using a $\mathrm{Ni}$ :Cr alloy tip positioned on the top of the device. Additionally, homogeneous sensing experiments are carried out with solutions in static mode by depositing droplets on the microresonator surface.

Figure 4. $a$ Resonances transmissions spectrum for racetrack microresonator and $b$ its corresponding normalized fast Fourier transform (FFT).

Figure 5. $a$ Resonance peak shifts for six thermal input values with air as upper cladding, and $b$ their corresponding linear responses for ascending and descending thermal input intensities.

Figure 6. $a$ Blue-shift on the normalized resonance peaks for six thermal input values, the racetrack microresonator being immersed in DI water $b$ Ascending and descending responses by respectively increasing and diminishing the thermal input intensities.

Figure 7. $a$ Resonance wavelength shifts of the racetrack microresonator based on UV210 photoresist immersed in glucose solutions with various concentration values. $b$ Reproducibility on the linear variations of resonance wavelength shift as a function of glucose concentrations. 


\section{Figure and table captions}

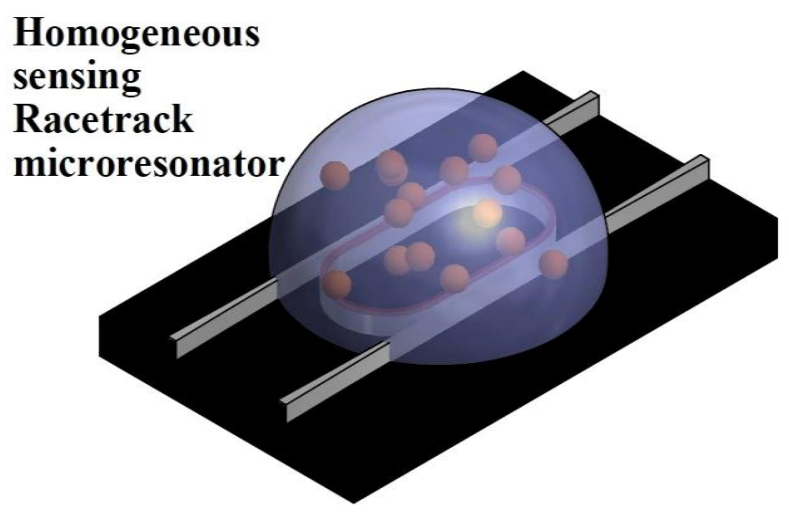

Abstract graphic

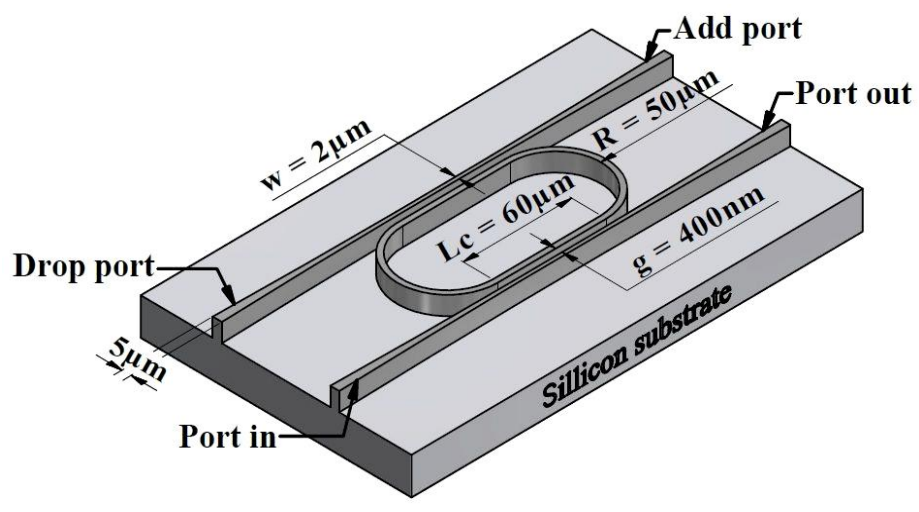

Figure 1 

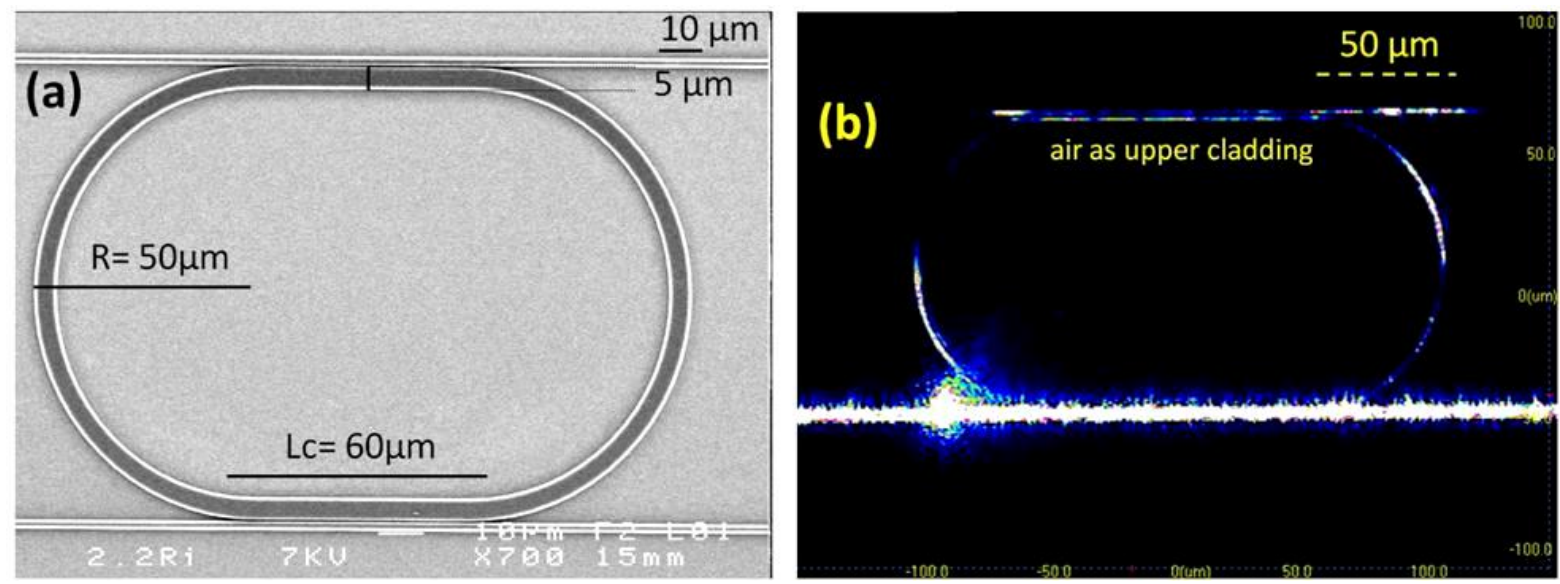

Figure 2

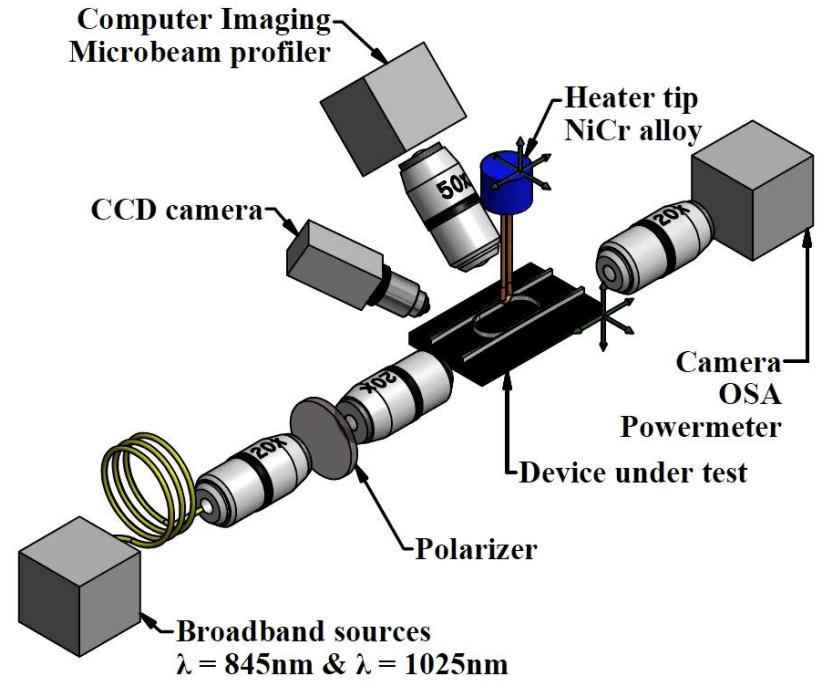

Figure 3 

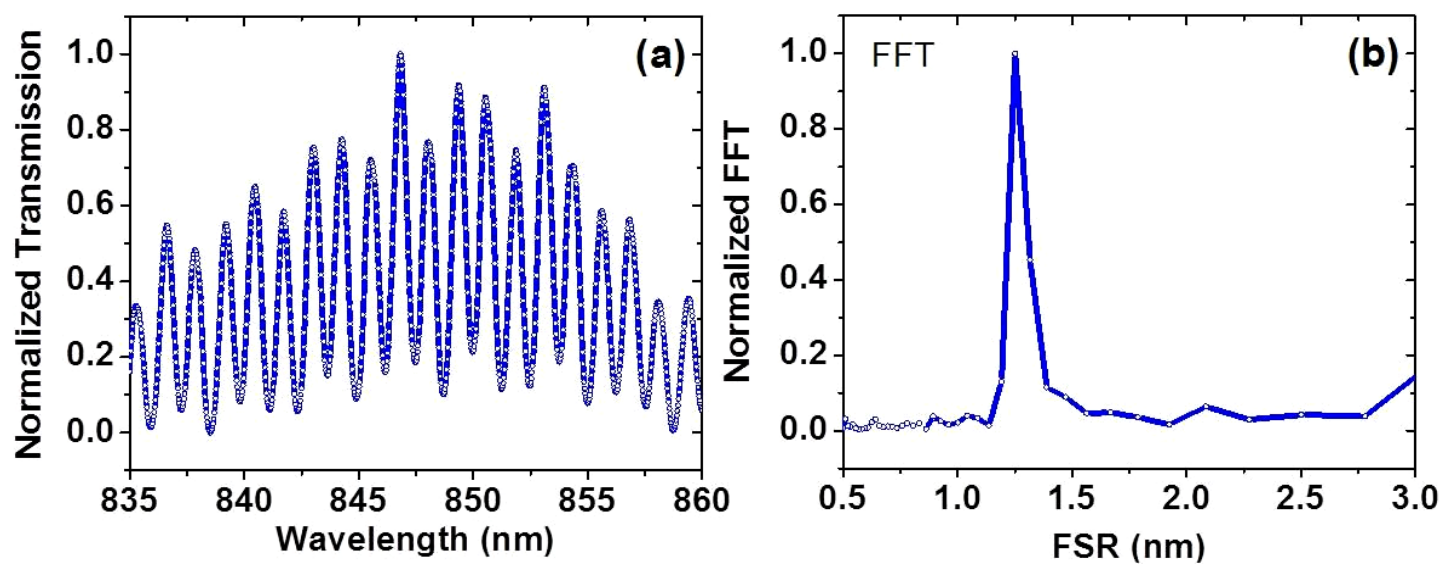

Figure 4
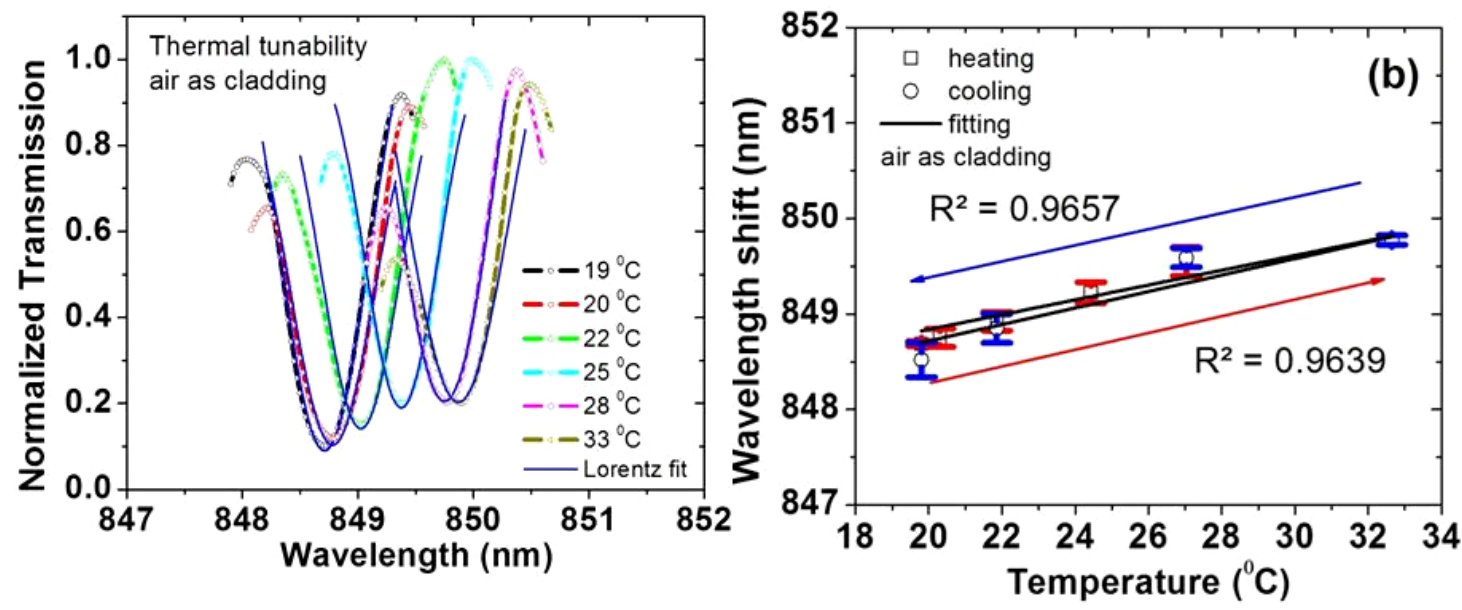

Figure 5 

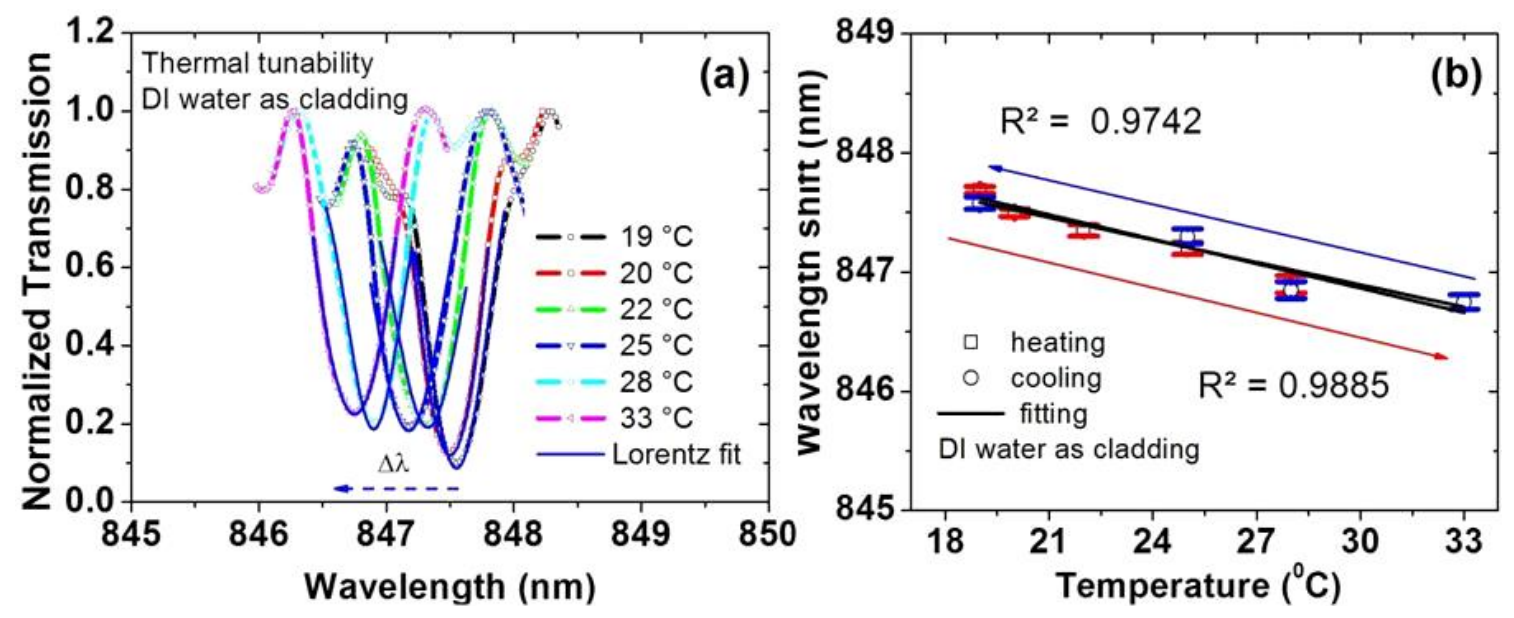

Figure 6
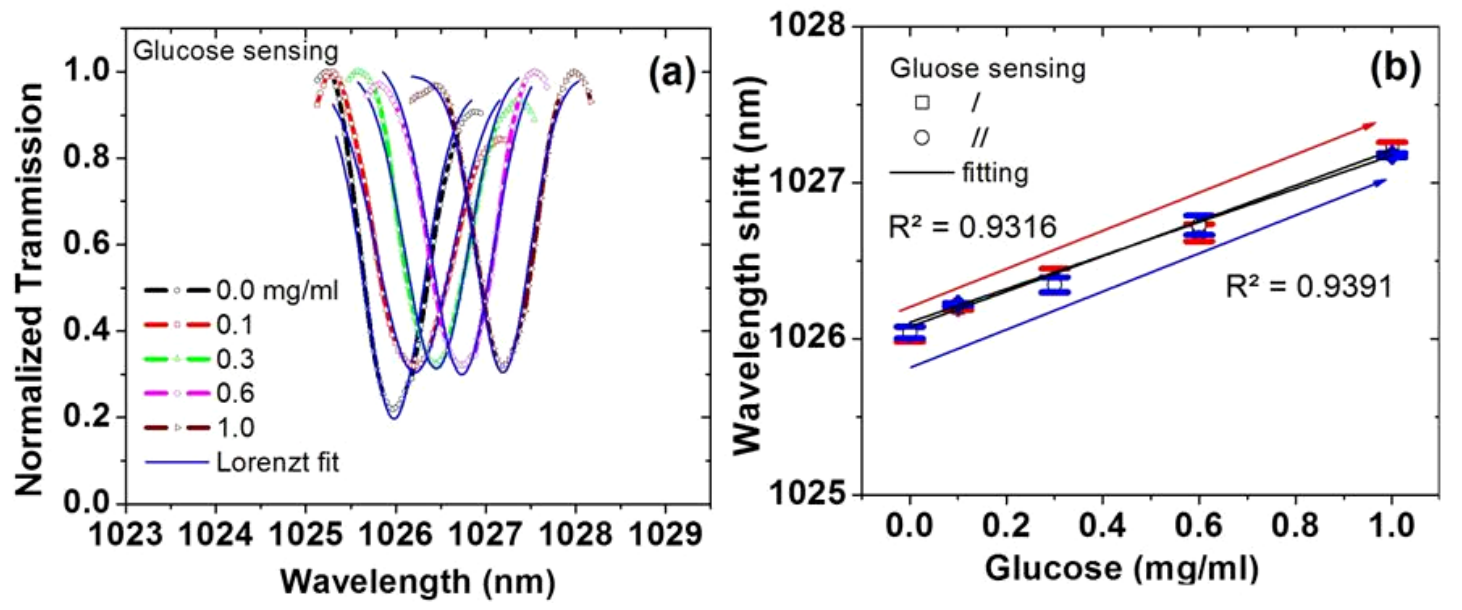

Figure 7 